\title{
Alteraçōes na Gasometria de Fetos Aloimunizados após Procedimento de Transfusão Intra-uterina
}

\author{
Fetal Blood Gas Changes in Red-cell Transfusion in Alloimmunization \\ Roseli Mieko Yamamoto Nomura, Estela Naomi Nishie, Maria de Lourdes Brizot, \\ Rossana Pulcinelli Vieira Francisco, Adolfo Wenjaw Liao, Marcelo Zugaib
}

\begin{abstract}
RESUMO
Objetivo: este estudo, realizado em gestações com aloimunização pelo fator $\mathrm{Rh}$, tem como objetivo descrever as alterações gasométricas e do equilíbrio ácido-básico fetal antes e após transfusões intra-uterinas (TIU).

Método: no periodo de junho de 2001 a outubro de 2001, antes e após a TIU em fetos de gestantes aloimunizadas, foram avaliados prospectivamente a gasometria e o equilíbrio ácido-básico no sangue da veia umbilical. As medidas foram realizadas em 8 amostras de sangue de 5 fetos. O sangue fetal foi obtido por cordocentese da veia umbilical antes e após TIU. Os resultados obtidos foram comparados com a expansão volêmica na TIU, a idade gestacional no procedimento, o peso fetal estimado pela ultra-sonografia e as variações da hemoglobina fetal $(g / d L)$.

Resultados: em todos os casos foi observada queda nos valores do $\mathrm{pH}$, com redução média de 0,09 (DP=0,02). A hemoglobina fetal apresentou aumento médio de 8,4 g/ dL (DP=2,9 g/ dL). Foi constatada também variação negativa da $\mathrm{pO}_{2}$ (média $=-1,28 \mathrm{mmHg}$ ) na concentração de $\mathrm{HCO}_{3}^{-}$(média = -2,25 mEq/l). Houve aumento da $\mathrm{pCO}_{2}$ (média $=3,2 \mathrm{mmHg}$ ) e redução nos valores do excesso de bases (média $=-3,75$ ).

Conclusão: a análise das gasometrias permite concluir que o procedimento de TIU acompanha-se de queda nos valores do $\mathrm{pH}$ de sangue de veia umbilical, demonstrando haver acidemia fetal relativa após o procedimento.
\end{abstract}

PALAVRAS-CHAVE: Aloimunização Rh. Transfusão intra-uterina. Gasometria fetal.

\section{Introdução}

A resposta fetal à hipoxia tem sido amplamente estudada em Obstetrícia, sendo o quadro clínico resultante desse processo denominado de sofrimento fetal. A deficiente troca de gases entre o produto conceptual e o organismo materno promove desequilíbrio no sistema ácido-básico fetal, cuja manifestação bioquímica é representada pela acidose fetal ${ }^{1}$. A gasometria tem sido empregada como principal método para aferir a condição de

Clínica Obstétrica do Hospital das Clínicas da Faculdade de Medicina da Universidade de São Paulo Correspondência:

Roseli Mieko Yamamoto Nomura

Rua General Canavarro, 280 - Bairro Campestre

09070-440 - Santo André - SP

Fone: (011) 4991-2481 - 4221-8778 FAX: 4221-8752

e-mail: roseli.nomura@terra.com.br acidose fetal, utilizando os valores do $\mathrm{pH}$ como parâmetro fundamental para esse diagnóstico ${ }^{2}$.

A doença hemolitica perinatal, na sua forma grave, tem como terapêutica a transfusão intra-uterina, que tem como objetivo a correção da anemia fetal. É quadro de comprometimento fetal grave, em que o sucesso da gestação está freqüentemente associado à correção da anemia fetal $^{3}$. A gestante com aloimunização pelo fator $\mathrm{Rh}(\mathrm{D})$ apresenta anticorpos circulantes anti-D, da classe IgG, que atravessam a barreira placentária e aderem às hemácias fetais, destruindo-as e provocando a anemia fetal. A gravidade da doença é determinada pela quantidade de anticorpos maternos que atravessam a placenta bem como pela sua afinidade pelo antígeno presente nas hemácias fetais $^{3}$. O feto desencadeia mecanismos de proteção da sua circulação, pois a anemia reduz a ca- 
pacidade de transporte de oxigênio aos tecidos, desencadeando respostas metabólicas fetais ${ }^{4}$.

A realização de procedimentos invasivos em casos de aloimunização para a realização da transfusão intra-uterina permite também a avaliação do equilíbrio ácido-básico fetal pela análise do sangue obtido por cordocentese. São poucos os estudos disponiveis até o momento que relatem as alterações dos parâmetros gasométricos em procedimento de transfusão intra-uterina na aloimunização fetal ${ }^{5}$.

A transfusão intra-uterina tem como objetivo principal a correção da anemia fetal e normalização das condições hemodinâmicas que permitem transporte adequado de oxigênio pelo organismo fetal ${ }^{4}$. Soothill et al. ${ }^{6}$ observam redução no conteúdo de $\mathrm{O}_{2}$ quando a concentração de hemoglobina fetal é menor que 4,0 g/dL, com aumento significativo na concentração de lactato, que corresponde à acidose metabólica fetal. A transfusão, ao provocar sobrecarga no feto com a expansão de sua volemia, poderá acarretar alterações no metabolismo do organismo fetal ${ }^{7}$.

Pouco se tem pesquisado sobre as alterações metabólicas e da gasometria fetais que acompanham esses procedimentos invasivos. Este estudo, realizado em gestações com aloimunização pelo fator $\mathrm{Rh}$, tem como objetivo descrever as alterações do equilíbrio ácido-básico em fetos que necessitam de transfusão intra-uterina e as alterações da gasometria fetal decorrentes do procedimento.

\section{Pacientes e Métodos}

Este estudo prospectivo foi realizado na Clínica Obstétrica do Hospital das Clínicas da Faculdade de Medicina da Universidade de São Paulo (HCFMUSP) no período de junho de 2001 a outubro de 2001. Foram analisados os dados de amostras coletadas em oito procedimentos de transfusão intra-uterina que foram realizados em um total de cinco fetos.

Foram utilizados os seguintes critérios de inclusão: diagnóstico de aloimunização pelo fator $\mathrm{Rh}$ na forma grave, com indicação para transfusão intra-uterina; acompanhamento pré-natal realizado na Clínica Obstétrica; gasometria realizada pelos pesquisadores envolvidos no projeto; gestação única; recém-nascido com ausência de anomalias estruturais ou cromossômicas fetais. Foram excluídos os casos em que a amostra apresentou-se coagulada no momento da análise gasométrica ou quando houve impossibilidade de coleta em quantidade suficiente para análise de todos os parâmetros estudados.

O sangue fetal foi obtido por cordocentese da veia umbilical utilizando agulha calibre 20 sob anestesia local e guiada por ultra-sonografia. Imediatamente antes do início da transfusão, foi coletada amostra de 0,5 $\mathrm{mL}$ em seringa heparinizada para a análise gasométrica antes do procedimento. Ao término da transfusão do volume sangüíneo programado, nova amostra para gasometria foi coletada da veia umbilical. As medidas foram realizadas imediatamente após a obtenção de cada amostra, utilizando-se equipamento de análise portátil I-STAT (Abbott Laboratórios do Brasil Ltda).

Foram medidos o $\mathrm{pH}$ e o excesso de bases (BE) das amostras obtidas do sangue fetal e os seguintes parâmetros gasométricos: pressão parcial de oxigênio $\left(\mathrm{pO}_{2}\right)$, pressão parcial de $\mathrm{CO}_{2}\left(\mathrm{pCO}_{2}\right)$, concentração de bicarbonato $\left(\mathrm{HCO}_{3}^{-}\right)$e saturação de $\mathrm{O}_{2}\left(\mathrm{SO}_{2}\right)$.

A mensuração da concentração de hemoglobina $(\mathrm{Hb})$ fetal antes e após a transfusão intra-uterina é exame de rotina para a realização do procedimento. $\mathrm{O}$ volume de sangue necessário para corrigir a anemia fetal é calculado considerando-se a hemoglobina fetal prévia, a hemoglobina do sangue a ser transfundido, a hemoglobina desejada pós-transfusão e o volume fetoplacentário médio para a idade gestacional ${ }^{8}$. Dessa forma foi calculado o volume de concentrado de hemácias a ser infundido e a percentagem de expansão da volemia fetal.

Foram analisados os resultados dos exames para a comparação entre as gasometrias realizadas antes e após a transfusão intra-uterina. Os parâmetros foram analisados descritivamente de forma que, para as variáveis quantitativas, foram calculadas as médias e desvios padrão.

Este estudo foi aprovado pela Comissão de Ética para Análise de Projetos de Pesquisa (CAPPesq) do HC-FMUSP, projeto número 753/01.

\section{Resultados}

Na Tabela 1 estão descritos os valores do $\mathrm{pH}$ pré e pós-transfusionais de todos os casos analisados, bem como os valores da hemoglobina fetal e a percentagem de expansão da volemia. Em todos os casos foi observada queda dos valores do $\mathrm{pH}$, cuja redução apresentou média de 0,09, com desvio padrão de 0,02. Em todas as transfusões houve incremento da hemoglobina fetal, corrigindo-se a anemia do feto e provocando expansão de sua volemia de $57,8 \%$ em média. A variação do aumento da hemoglobina apresentou média de 8,4 $\mathrm{g} / \mathrm{dL}$ com desvio-padrão de $2,9 \mathrm{~g} / \mathrm{dL}$. 


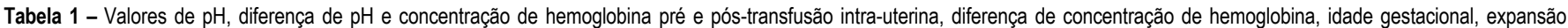
da volemia fetal e peso estimado fetal em 8 transfusões.

\begin{tabular}{|c|c|c|c|c|c|c|c|c|c|}
\hline Caso & IG (sem) & Exp. (\%) & Peso fetal (g) & pH pré & pH pós & $\Delta \mathrm{pH}$ & Hb pré $(g / d L)$ & Hb pós (g/dL) & $\Delta \mathrm{Hb}(\mathrm{g} / \mathrm{dl})$ \\
\hline 11 & $28 s 6 d$ & 53,9 & 1.517 & 7,39 & 7,29 & $-0,10$ & 6,1 & 14,8 & 8,7 \\
\hline 2 & $32 s 2 d$ & 62,7 & 2.349 & 7,40 & 7,33 & $-0,07$ & 10,1 & 15,8 & 5,7 \\
\hline 21 & $31 \mathrm{~s} 3 \mathrm{~d}$ & 68,7 & 1.766 & 7,42 & 7,33 & $-0,09$ & 6,9 & 17,6 & 10,7 \\
\hline 2 & $34 s 3 d$ & 34,1 & 2.454 & 7,40 & 7,33 & $-0,06$ & 10,5 & 14,3 & 3,8 \\
\hline 31 & $20 s 3 d$ & 57,3 & 336 & 7,42 & 7,32 & $-0,11$ & 8,7 & 18,7 & 10 \\
\hline 2 & $31 \mathrm{~s} 1 \mathrm{~d}$ & 50,0 & 1.597 & 7,35 & 7,29 & $-0,06$ & 9,2 & 18,1 & 8,9 \\
\hline 4 & $30 s 5 d$ & 67,6 & 1.825 & 7,38 & 7,31 & $-0,07$ & 9,5 & 16,1 & 6,6 \\
\hline 5 & $21 s 6 d$ & 68,0 & 480 & 7,42 & 7,28 & $-0,14$ & 3,7 & 16,4 & 12,7 \\
\hline Média & $28 s 6 d$ & 57,8 & $1.540,5$ & 7,40 & 7,31 & $-0,09$ & 8,1 & 16,5 & 8,4 \\
\hline
\end{tabular}

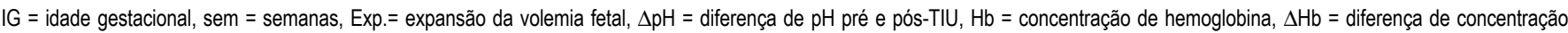
de hemoglobina pré e pós-TIU.

As alterações das concentrações dos gases no sangue fetais são apresentadas na Tabela 2, onde se pode verificar que em seis de sete transfusões intra-uterinas analisadas houve queda da $\mathrm{pO}_{2}$ fetal. Em relação à concentração de $\mathrm{pCO}_{2}$, observou-se aumento médio de 3,2 $\mathrm{mmHg}$, com desvio padrão de $2,2 \mathrm{mmHg}$.

$\mathrm{Na}$ Tabela 3 estão as variações ocorridas na concentração de bicarbonato decorrentes da transfusão intra-uterina. Foi observado, em todos os casos, redução na concentração de bicarbonato, com variação média de $2,25 \mathrm{mEq} / \mathrm{L}$ e desvio padrão de 1,28 $\mathrm{mEq} / \mathrm{L}$. As alterações observadas no BE demonstram aumento deste parâmetro em todos os casos, com variação média de 3,75 e desvio padrão de 1,49, correspondendo ao maior consumo de bases tampões.

Tabela 2 - Valores de $\mathrm{pO}_{2}$ em mmHg, diferença entre $\mathrm{pO}_{2}, \mathrm{pCO}_{2}$ e diferença de $\mathrm{pCO}_{2}$ pré e pós-TIU.

\begin{tabular}{|c|c|c|c|c|c|c|c|}
\hline \multirow{2}{*}{ Caso 1} & & \multirow{2}{*}{$\begin{array}{c}\mathrm{pO}_{2} \text { pré } \\
38\end{array}$} & \multirow{2}{*}{$\begin{array}{c}\mathrm{pO}_{2} \text { pós } \\
33\end{array}$} & \multirow{2}{*}{$\begin{array}{c}\Delta \mathrm{pO}_{2} \\
-5\end{array}$} & \multirow{2}{*}{$\begin{array}{c}\mathrm{pCO}_{2} \\
34,5\end{array}$} & \multirow{2}{*}{$\frac{\mathrm{pCO}_{2}}{40,5}$} & \multirow{2}{*}{$\frac{\Delta \mathrm{pCO}_{2}}{6}$} \\
\hline & 1 & & & & & & \\
\hline & 2 & 42 & 41 & -1 & 38,5 & 39,9 & 1,4 \\
\hline Caso 2 & 1 & 37 & 35 & -2 & 33,2 & 39 & 5,8 \\
\hline & 2 & 31 & 26 & -5 & 36,7 & 41,3 & 4,6 \\
\hline Caso 3 & 1 & 56 & 54 & -2 & 27,2 & 30,7 & 3,5 \\
\hline & 2 & - & - & - & 34,7 & 35,4 & 0,7 \\
\hline Caso 4 & & 44 & 42 & -2 & 36,3 & 36,9 & 0,6 \\
\hline Caso 5 & & 44 & 52 & 8 & 36,2 & 39,2 & 3,0 \\
\hline Média & & 41,7 & 40,4 & $-1,3$ & 34,7 & 37,9 & 3,2 \\
\hline
\end{tabular}

$\Delta \mathrm{pO}_{2}=$ diferença de $\mathrm{pO}_{2}$ pré e pós-TIU, $\Delta \mathrm{pCO}_{2}=$ diferença de $\mathrm{pCO}_{2}$ pré e pós-TIU.

Tabela 3 - Valores de $\mathrm{HCO}_{3}^{-}(\mathrm{mEq} / \mathrm{l})$, diferença entre $\mathrm{HCO}_{3}^{-}$pré e pós-TIU; BE e diferença de BE pré e pós-TIU.

\begin{tabular}{|c|c|c|c|c|c|c|c|}
\hline & & $\mathrm{HCO}_{3}^{-}$pré & $\mathrm{HCO}_{3}^{-}$pós & $\triangle \mathrm{HCO}_{3}^{-}$ & BE pré & BE pós & $\Delta \mathrm{BE}$ \\
\hline \multirow[t]{2}{*}{ Caso 1} & 1 & 21 & 19 & -2 & -4 & -7 & -3 \\
\hline & 2 & 24 & 21 & -3 & -1 & -5 & -4 \\
\hline \multirow[t]{2}{*}{ Caso 2} & 1 & 21 & 20 & -1 & -3 & -6 & -3 \\
\hline & 2 & 23 & 22 & -1 & -2 & -4 & -2 \\
\hline \multirow[t]{2}{*}{ Caso 3} & 1 & 18 & 16 & -2 & -7 & -11 & -4 \\
\hline & 2 & 19 & 17 & -2 & -7 & -10 & -3 \\
\hline Caso 4 & & 21 & 19 & -2 & -4 & -8 & -4 \\
\hline Caso 5 & & 23 & 18 & -5 & -1 & -8 & -7 \\
\hline Média & & 21,3 & 19 & $-2,3$ & $-3,6$ & $-7,4$ & $-3,8$ \\
\hline
\end{tabular}

$\Delta \mathrm{HCO}^{3}=$ diferença de $\mathrm{HCO}_{3}{ }^{-}$pré e pós-TIU, $\triangle \mathrm{BE}=$ diferença de excesso de bases pré e pós-TIU. 
Quanto aos resultados perinatais, em quatro casos foi realizada a operação cesariana e um caso evoluiu para parto vaginal. Apenas uma gestação atingiu o termo, com idade gestacional no nascimento de 37 semanas e 1 dia. As demais tiveram suas gestações concluídas entre 36 e 37 semanas de gestação. Todos os recém-nascidos apresentaram peso adequado para a idade gestacional e a média do peso no nascimento foi de 2.742 gramas. $\mathrm{O}$ indice de Apgar de primeiro minuto demonstrou valor inferior a $7 \mathrm{em}$ dois recém-nascidos e o de quinto minuto foi normal $(>7)$ em todos os casos. Não houve nenhum caso de óbito neonatal.

\section{Discussão}

A hemoglobina fetal é responsável pelo transporte de oxigênio no sistema circulatório e desempenha também importante papel no sistema tampão do sangue humano. Alguns estudos demonstram não haver alterações no $\mathrm{pH}$ e na concentração dos gases no sangue da veia umbilical em fetos com anemia moderada ${ }^{1-3}$. Já a análise de casos de aloimunização com hidropisia fetal e anemia grave, antes da transfusão intra-uterina, mostra correlação significativa entre a concentração de hemoglobina e o pH, a concentração de bicarbonato e o excesso de bases da artéria umbilical ${ }^{6}$. A anemia fetal grave é situação em que o feto está sujeito à hipoxia anêmica, e quando o organismo fetal não consegue manter a oxigenação tecidual pelos mecanismos compensatórios, pode se instalar a acidemia.

No presente estudo observou-se queda no $\mathrm{pH}$ fetal após a transfusão, em conformidade com os dados descritos por Vandenbussche et al. ${ }^{5}$, que observam queda de, em média, 0,05 no valor do $\mathrm{pH}$. Entretanto, esses autores não realizam avaliação das alterações no excesso de bases que, no presente estudo, demonstrou ser parâmetro que apresenta variação com a transfusão por maior consumo das bases tampões.

$\mathrm{Na}$ análise das gasometrias verificou-se que o procedimento de transfusão intra-uterina foi acompanhado de queda nos valores do $\mathrm{pH}$ do sangue da veia umbilical fetal, assim como dos valores de $\mathrm{pO}_{2}$ e aumento das concentrações de $\mathrm{pCO}_{2}$, indicando haver acidemia e hipoxemia relativas após o procedimento ${ }^{9}$. Da mesma maneira, Nicolini et al. ${ }^{10}$ encontraram um aumento estatisticamente significativo na concentração de $\mathrm{pCO}_{2}$ após a transfusão de sangue, provavelmente devido às altas concentrações de $\mathrm{pCO}_{2}$ no sangue doador. As alterações desses parâmetros podem estar relacionadas ao sangue utilizado na transfusão ${ }^{11}$ e são aspectos que merecem ser analisados quando o procedimento é indicado. Em um caso foi realizada a análise do $\mathrm{pH}$ do sangue a ser transfundido e constatou-se $\mathrm{pH}$ de 6,59. Considerando-se que a expansão volêmica é quase sempre superior a $50 \%$, podemos inferir que o baixo valor do $\mathrm{pH}$ do sangue usado na transfusão deve participar na etiologia da acidemia relativa observada após o procedimento. A análise do $\mathrm{pH}$ do sangue a ser transfundido é aspecto de interesse para futuras pesquisas, nas quais será possivel verificar o real valor dessa análise na avaliação da qualidade do sangue utilizado.

A queda na $\mathrm{pO}_{2}$ fetal após o procedimento é de pequena magnitude e não chega a comprometer a oxigenação fetal. Pode-se constatar que a $\mathrm{pO}_{2}$ após a transfusão intra-uterina se mantém dentro de limites normais, pois, provavelmente, as trocas gasosas no território placentário permaneceram preservadas. $\mathrm{O} \mathrm{CO}_{2}$, por ser gás de elevada capacidade de difusão pela barreira placentária, também mostrou pequena variação nas suas concentrações.

A variação negativa nas concentrações de bicarbonato ocorrem pela mobilização deste sistema tampão no processo de acidemia relativa observada com o procedimento da transfusão intrauterina. A variação negativa no excesso de bases também demonstra a mobilização dos sistemas tampões. Entretanto, em nenhum caso aqui analisado o valor do $\mathrm{BE}$ atingiu nivel patológico inferior a -12 . $\mathrm{O}$ equivalente se constata na variação do $\mathrm{pH}$, pois, apesar da acidemia relativa ao nivel pré-transfusional, o valor absoluto do $\mathrm{pH}$ pós transfusão intra-uterina não atinge valores inferiores a 7,20 , o que caracterizaria a acidemia relacionada ao sofrimento fetal.

Outro aspecto que interessa na transfusão intra-uterina é o estado metabólico fetal no momento do procedimento ${ }^{10}$. A transfusão realizada em fetos que já apresentem acidemia provocará redução ainda maior do $\mathrm{pH}$ e, na dependência da magnitude dessa alteração, efeitos deletérios da acidose tecidual poderão ocorrer. Portanto, a programação da transfusão intra-uterina deverá ponderar esses aspectos, prevendo que a indicação oportuna do procedimento deverá atentar para que o feto ainda não tenha desencadeado as alterações metabólicas do sofrimento e acidose fetal.

O conhecimento do estado metabólico fetal permite melhor compreensão da resposta fetal ao procedimento transfusional e possibilitará planejamento adequado da expansão volêmica a ser programada $^{12}$. É freqüente a observação de alterações nos padrões da freqüência cardiaca fetal (FCF) imediatamente após a transfusão intra-uterina, 
e isso deve decorrer do uso de substâncias curarizantes associado às alterações metabólicas desencadeadas. O retorno da homeostase fetal demandará certo período de tempo, e isso é observado quando é realizada a monitorização fetal após o procedimento, podendo demorar até duas horas para que apresente padrões normais da $\mathrm{FCF}^{3}$.

O conhecimento das alterações metabólicas e gasométricas na transfusão intra-uterina contribui no planejamento do procedimento e na avaliação da resposta cardíaca fetal ${ }^{13,14}$. O presente estudo demonstra ocorrer queda nos valores do $\mathrm{pH}$ fetal após a transfusão intra-uterina em gestações com aloimunização pelo fator $\mathrm{Rh}$ e deve ser aspecto a ser ponderado quando o procedimento é indicado.

ABSTRACT

Purpose: the aim was to study Rh-alloimmunized pregnant women and describe the gasometric abnormalities and the fetal acid-base changes before and after intrauterine transfusion.

Methods: between June 2001 and October 2001, before and after intrauterine transfusion in the alloimunized fetuses, gasometric data and acid-base parameters were prospectively studied in the umbilical vein blood. The measurements were performed in 8 samples of 5 fetuses. The fetal blood was obtained by cordocentesis before and after the intrauterine transfusion. The results were compared to the volemic expansion, the gestational age at procedure, the estimated fetal weight and the hemoglobin values $(g / d L)$.

Results: all the cases showed $p H$ value reduction, mean of $0.09(S D=0.02)$. The fetal hemoglobin value showed a mean improvement of $8.4 \mathrm{~g} / \mathrm{dL}(\mathrm{SD}=2.9 \mathrm{~g} / \mathrm{dL})$. The $\mathrm{pO}_{2}$ and $\mathrm{HCO}_{3}$ concentrations showed negative variation (mean $\triangle \mathrm{pO}_{2}=$ $1.28 \mathrm{mmHg}$, mean $\left.\triangle \mathrm{HCO}_{3}^{-}=-2.25 \mathrm{mEq} / \mathrm{L}\right) . p \mathrm{CO}_{2}$ showed improvement (mean $\triangle p C \mathrm{O}_{2}=3.2 \mathrm{mmHg}$ ) and reduced values of base excess occurred (mean $=-3.75$ ).

Conclusion: the gasometric analysis allows to conclude that intrauterine transfusion is followed by $\mathrm{pH}$ reduction in the umbilical vein, with relative fetal acidemia after the procedure.

KEYWORDS: Rh alloimunization. Intrauterine transfusion. Fetal gasometry.

\section{Referências}

1. Goldaber KG, Gilstrap LC 3rd. Correlations between obstetric clinical events and umbilical cord blood acid-base and blood gas values. Clin Obstet Gynecol 1993; 36:47-59.
2. Francisco RPV, Yamamoto RM, Miyadahira S, Cunha CL, Zugaib M. Correlação entre testes para avaliação da vitalidade fetal, $\mathrm{pH}$ da artéria umbilical e os resultados neonatais em gestações de alto risco. Rev Bras Ginecol Obstet 2000; 22:503-10.

3. Farina A, Calderoni P, Simonazzi G, Carinci P, Rizzo N. Survival analysis of transfused fetuses affected by Rh-alloimunization. Prenat Diagn 2000; 20:881-5.

4. Klumper FJ, van Kamp IL, Vandenbussche FP, et al. Benefits and risks of fetal red-cell transfusion after 32 weeks gestation. Eur J Obstet Gynecol Reprod Biol 2000; 92:91-6.

5. Vandenbussche FP, van Kamp IL, Oepkes D, Hermans J, Bennebroek Gravenhorst J, Kanhai HH. Blood gas and $\mathrm{pH}$ in the human fetus with severe anemia. Fetal Diagn Ther 1998; 13:115-22.

6. Soothill PW, Nicolaides $\mathrm{KH}$, Rodeck $\mathrm{CH}$. Effect of anaemia on fetal acid-base status. $\mathrm{Br} \mathrm{J}$ Obstet Gynaecol 1987; 94:880-3.

7. Moise KJ Jr. Management of rhesus alloimmunization in pregnancy. Obstet Gynecol 2002; 100:600-11.

8. Nicolaides KH, Clewell WH, Rodeck CH. Measurement of human fetoplacental blood volume in erythroblastosis fetalis. Am J Obstet Gynecol 1987; 157:50-3.

9. Westgren $M$, Lingman $G$, Stangenberg $M$. Oxygenation of human fetus as a function of hemoglobin concentration. Am J Perinatol 1994; 11:9-13.

10.Nicolini U, Santolaya J, Fisk NM, et al. Changes in fetal acid-base status during intravascular transfusion. Arch Dis Child 1988; 63:710-4.

11.Weiner CP, Williamson RA, Wenstrom K, et al. Management of fetal hemolytic disease by cordocentesis. II. Outcome of treatment. Am J Obstet Gynecol 1991; 165:1302-7.

12. Noia G, De Santis M, Romano D, et al. Complementary therapy for severe Rhalloimmunization. Clin Exp Obstet Gynecol 2002; 29:297-301.

13. Radunovic N, Lockwood CJ, Alvarez M, Plecas D, Chitkara U, Berkowitz RL. The severely anemic and hydropic isoimmune fetus: changes in fetal hematocrit associated with intrauterine death. Obstet Gynecol 1992; 79:390-3.

14.Westgren M, Selbing A, Stangenberg M, Phillips R. Acid-base status in fetal heart blood in erythroblastotic fetuses: a study with special reference to the effect of transfusions with adult blood. Am J Obstet Gynecol 1989; 160:1134-8.

\section{Conflitos de interesses}

O aparelho I-STAT para a realização da pesquisa foi cedido pela Abbott do Brasil. 